\title{
Emergencia del modelo de enfermería transmitido en las universidades españolas: una aproximación analítica a través de la Teoría Fundamentada
}

\author{
Emergence of the nursing model transmitted in Spanish \\ universities: an analytical approach through Grounded Theory
}

Olga Rodrigo Pedrosa ${ }^{1}$

Jordi Caïs ${ }^{2}$

Cristina Monforte-Royo ${ }^{3}$
${ }^{1}$ Escuela Universitaria de Enfermería Sant Joan de Déu, Universidad de Barcelona. Calle Miret i Sans 10-16, Esplugues de Llobrega. 08034 Barcelona Catalunha Espanha. orodrigo@ santjoandedeu.edu.es ${ }^{2}$ Departamento de Sociología de las Profesiones, Universidad de Barcelona. Barcelona

Catalunha Espanha.

${ }^{3}$ Departamento de

Enfermería, Universitat Internacional de Catalunya. Barcelona Catalunha Espanha.

\begin{abstract}
The scope of this paper was to ascertain the meaning of the term "nursing" among teaching nurses at Spanish universities. The research was qualitative, and the strategy used was analytical induction. Sampling was theoretical and focused on the selection of extreme cases and the data collection technique was in-depth interviews. The analysis was carried out using the specific Grounded Theory technique. Strategies were established to ensure credibility, portability, confirmability and auditability. Based on the analysis of the data, three categories were identified that responded to the planned objective: it is a "human activity linked to motherhood," a "vocation to help others that some people have" and an "activity that takes care of the needs of others respecting their identity." It can be concluded that in Spanish universities a model of nurse is being transmitted where the proper role of care is inherent to the feminine gender and the vocation of service to others that some people have. This is the reason why the Spanish Royal Academy (RAE) only identifies the role of technical assistance to medicine as a professional element of nursing.

Key words Gender identity, Grounded theory, Nursing care, Nursing theory, Vocational orientation
\end{abstract}

Resumen El objetivo fue conocer el significado del término "enfermería" para las enfermeras docentes de las universidades españolas. El diseño fue cualitativo. Como estrategia se utilizó la inducción analítica. El muestreo fue teórico y enfocado en la selección de casos extremos. La técnica de recogida de datos fue la entrevista en profundidad. El análisis se llevó a cabo mediante la técnica específica de la Teoría Fundamentada. Se establecieron estrategias para asegurar la credibilidad, transferibilidad, confirmabilidad y auditabilidad. A partir del análisis de los datos se identificaron tres categorías que dieron respuesta al objetivo planteado: es una "actividad humana vinculada a la maternidad", una "vocación de ayuda al otro que tienen algunas personas" y una "actividad que atiende las necesidades de los demás respetando su identidad". Se puede concluir que en las universidades españolas se está transmitiendo un modelo de enfermera donde el rol propio de cuidado es inherente al género femenino y a la vocación de servicio al otro que tienen algunas personas. Esto es causa de que la Real Academia de la Lengua Española sólo identifique el rol de asistencia técnica a la medicina como elemento profesional de la enfermería.

Palabras clave Atención de enfermería, Identidad de género, Orientación vocacional, Teoría de enfermería, Teoría fundamentada 


\section{Introducción}

En términos generales se ha definido "profesión" como una ocupación que monopoliza una serie de actividades privadas sobre la base de un gran acervo de conocimiento abstracto. Esto permite a quien lo desempeña una considerable libertad de acción que tiene importantes consecuencias sociales ${ }^{1}$. Para Rodríguez ${ }^{2}$ las profesiones tienen que estar orientadas hacia el bienestar de la sociedad tratando de ser una contribución única y específica de mejora y progreso. En cuanto a la enfermería como profesión sus orígenes se atribuyen a la enfermera inglesa Florence Nigthingale (1820-1910) $)^{3,4}$ quien la define cómo: algo más que administrar medicinas y cataplasmas [...]. Es poner al enfermo en las mejores condiciones para que la naturaleza actúe $e^{5}$. Sin embargo en el caso español el inicio de la profesionalización de la enfermería se debe a la iniciativa de un médico, el Dr. Federico Rubió y Galí, quien en 1986 crea la primera escuela de enfermería ${ }^{6}$ con el objetivo de conseguir enfermeras capaces de aprender cuanto corresponda al arte de atender y cuidar de modo artístico y científico [...] para ganarse la vida asistiendo enfermos ${ }^{6}$. Cuando se institucionaliza la profesión en España se establece como su rol primordial el ayudar al médico a cumplir puntualmente lo que éste ordene $e^{7}$. Sin embargo el modelo de enfermera propuesto por Nigthingale, pese a que ésta también limitó la actividad al control explícito de la medicina ${ }^{8}$, orientó su atención a la devoción al paciente ${ }^{8}$ y a su cuidado . $^{8}$

A inicios de los años setenta del siglo XX se produce, entre varios grupos de enfermeras españolas, un interés por promover la enfermería a rango de disciplina científica influenciada por el movimiento enfermero iniciado en Estados Unidos y Canadá ${ }^{10}$, modelado a partir de Nigthingale 9 . Durante esta época la llegada a España de libros sobre modelos conceptuales de enfermería generados en los años 50 del siglo XX en Norteamérica $^{11}$, la nueva visión que del trabajo de enfermería traían los profesionales que habían tenido la oportunidad de ejercer en los países anglo-americanos, junto al mayor aperturismo del país que conlleva la transición política hacia un estado democrático a partir de la muerte del general Franco en 1975, propició que comenzaran a surgir grupos de enfermeras que en palabras de Santo-Tomas (2000) "empezaran a plantearse la búsqueda de su propia identidad"3. Como consecuencia de ello en 1977, y tras multitudinarias movilizaciones, en forma de encierros y manifestaciones por parte del colectivo en todas la provincias españolas ${ }^{4}$, se aprueba mediante el Real Decreto 2128/77, la integración en la universidad de los estudios de enfermería bajo la titulación de Diplomado Universitario en Enfermería $(\mathrm{DUE})^{12}$. El nuevo plan de estudios, en sintonía con las corrientes de pensamiento anglo-americanas basadas en el modelo de Nigthingale, supone un cambio de orientación profesional de la asistencia técnica a la medicina que había tenido históricamente hasta ese momento ${ }^{3,10,13,14}$, a uno nuevo centrado en el cuidado de la persona ${ }^{3}$. A partir de ese momento $y$, por primera vez en la historia se reconoce a la enfermería española como profesión una contribución única y específica a la sociedad ${ }^{2}$ al margen de la disciplina médica: el proporcionar "cuidados profesionales enfermeros" 10 .

Otra novedad importante es la incorporación de las enfermeras como docentes en las universidades españolas mediante la Orden del 13 de diciembre de $1978^{15}$. Hasta ese momento las enfermeras habían sido formadas fundamentalmente por los médicos en las Facultades de Medicina. A partir de ese momento las enfermeras empiezan a responsabilizarse de determinadas asignaturas que tenían en su denominación la palabra Enfermería y, especialmente, de la asignatura de Enfermería Fundamental ${ }^{3}$. Esta asignatura, inédita en los planes de estudio de la enfermería hasta ese momento en España, incluía los contenidos específicos para poder llevar a la práctica el nuevo rol profesional ${ }^{3,16}$ en forma de Teorías y Modelos enfermeros, Historia de la profesión, Deontología Profesional, Cuidados Básicos y Metodología Enfermera ${ }^{4}$. Por primera vez en España las enfermeras estudian de manera diferenciada los aspectos relacionados con su disciplina y profesión, así como los cuidados llevados a cabo a través de un método ${ }^{4}$.

Actualmente, y después de casi 40 años con el nuevo enfoque profesional de la enfermería, la Real Academia de la Lengua Española (RAE) como autoridad lingüística, la sigue definiendo como profesión y titulación de la persona que se dedica al cuidado y atención a los enfermos y heridos, así como a otras tareas sanitarias, siguiendo pautas clínicas ${ }^{17}$. Y el término enfermera/o cómo persona dedicada a la asistencia a los enfermos ${ }^{18}$. Estos significados representan un modelo de profesión enfermera que se ajusta más al propio de asistente del médico que al modelo anglo-americano de orientación al cuidado de la persona ${ }^{19}$. En relación a esto para Whorf ${ }^{20}$, las lenguas no constituyen simplemente instrumentos de comunicación, sino que en sí mismas forman las 
ideas actuando como guía de la actividad mental del individuo, del análisis de sus impresiones y de la síntesis de todo lo que la mente hubiera registrado. Según Atchinson ${ }^{21}$ el lenguaje es particularmente eficiente para distinguir roles sociales, mantener los vínculos sociales e influenciar otros. Tras lo expuesto, se identifica una disociación en el caso español entre el significado académico del término enfermería, representado por un plan de estudios enfocado al cuidado profesional basado en teorías y modelos anglo-americanos ${ }^{11} \mathrm{y}$, su significado social, en este caso representado por la definición del término propuesta por la RAE, que continua orientándolo a un rol social de asistencia técnica a la medicina. En relación a esto la identidad profesional no es un proceso consensuado, estable y unitario, si no el resultado de procesos de interacción social múltiples, emergentes y cambiantes, que a su vez tienen carácter temporal ${ }^{11,22}$.

\section{Objetivo}

El objetivo de este estudio es conocer el significado del término "enfermería” para las enfermeras que ejercen como docentes en las universidades españolas. Con ello se pretende indagar si, como individuos que forman parte del contexto sociohistórico español, continúan socializando a las nuevas generaciones de enfermeras en una identidad profesional centrada en el modelo de "asistente técnico del médico" que propone la RAE o, por el contrario, están transmitiendo una identidad profesional centrada en llevar a cabo "cuidados profesionales enfermeros" modelada a partir de las Teorías y Modelos enfermeros anglo-americanos.

\section{Método}

\section{Diseño}

El diseño fue cualitativo y como estrategia metodológica se utilizó la inducción analítica con el objetivo, mediante la selección intencional de casos extremos, del mayor ajuste posible entre los datos y la realidad que se pretende hacer emerger ${ }^{23,24}$. Se utilizó descripción interpretativa de entrevistas con los participantes ${ }^{25,26}$.

\section{Técnica de muestreo}

Para la selección de participantes se llevó a cabo un muestreo teórico de manera que el análisis de los datos aportados por cada participante guió la subsiguiente selección muestral ${ }^{24}$. El principal criterio de inclusión fue haber ejercido como enfermeras o enfermeros docentes en las universidades españolas un mínimo de 5 años. Se utilizó el criterio de suficiencia para establecer inicialmente el número de participantes a reclutar buscando la máxima variabilidad de contextos y experiencias ${ }^{27} \mathrm{y}$, posteriormente, el criterio de saturación teorética, para determinar el momento de finalizar su recogida al no aportar los datos información nueva ${ }^{28}$ relativa al significado del término "enfermería” para las participantes.

La muestra final está formada por 8 participantes todos ellos enfermeros docentes que ejercían en universidades españolas y que tenían la titulación de Diplomado en Enfermería (titulación mínima necesaria para ejercer como docente en enfermería en las Universidades españolas). Los seis primeros fueron reclutados en el muestro inicial y todos ellos habían ejercido previamente como enfermeras el ámbito asistencial. A partir del análisis de las entrevistas se identificó la necesidad de incorporar dos casos extremos, que finalmente no aportaron nueva información con lo cual se consideró alcanzada la saturación teórica. El primer caso extremo era una enfermera docente que no había ejercido en la asistencia si no en el ámbito de la gestión y, el segundo caso extremo, era un enfermero que había ejercido en el ámbito asistencial como miembro de una orden religiosa.

En relación a las características de los participantes el $75 \%(n=6)$ fueron mujeres frente al 25 $\%$ de hombres $(n=2)$. En cuanto a la tipología de universidad el 62,5\% $(n=5)$ trabajaban en instituciones privadas, el $25 \%(\mathrm{n}=2)$ en instituciones públicas y el 12,5\% $(\mathrm{n}=1)$ ejercían freelance. El 37,5\% $(\mathrm{n}=3)$ de las instituciones eran de carácter laico frente al 62,5\% $(n=5)$ eran de confesionalidad cristiana. En cuanto a los participantes el $75 \%(n=6)$ impartían la asignatura de "Enfermería Fundamental” y el $25 \%(n=2)$ la asignatura de "Administración de los Servicios de Enfermería”. Por último el 37,5 \% $(\mathrm{n}=3)$ de los participantes procedían de Cataluña, $25 \%$ (n = 2) de Andalucía, el 12,5\% de la Comunidad de Madrid $(\mathrm{n}=1)$, el $12,5 \%$ de la Comunidad Valenciana $(\mathrm{n}=1)$ y el $12,5 \%(\mathrm{n}=1)$ de las Islas Baleares. Las entrevistas fueron realizadas entre el año 2012 y 2015 y tuvieron una duración de entre 60 y 90 minutos. 


\section{Técnica de recogida de datos}

En cuanto a la técnica de recogida de datos se llevaron a cabo entrevistas en profundidad ${ }^{27}$ Para su diseño se siguieron las recomendaciones de Dolbeare \& Schuman ${ }^{27}$ planteando la exploración secuencial de tres temas con el objetivo de facilitar la inducción del significado que tiene el término "enfermería" para las enfermeras docentes a partir de su experiencia vivida. El primer tema planteado Explícame tu historia como enfermera/o docente tenía como objetivo situar la experiencia en el contexto histórico. El segundo tema Explícame cuál es tu experiencia actual como enfermera/o docente tenía como finalidad situar la experiencia en el contexto actual y el último tema Explícame que significa para ti esa experiencia vivida tenía como objetivo indagar el significado que esa experiencia ha tenido para la persona que la ha vivido situada en un contexto socio histórico concreto. Los participantes fueron reclutados a partir de contactos de los miembros del equipo de investigación todos ellos vinculados a Escuelas Universitarias de Enfermería del territorio español. Las entrevistas fueron realizadas por el investigador principal que se presentó a los participantes como profesora de enfermería. En un primer contacto por correo electrónico se hizo llegar a los participantes la información del proyecto solicitándose su participación. A partir de ahí se concertó una entrevista en persona en el lugar escogido por los participantes, - en todos los casos su lugar de trabajo -, donde se firmó el consentimiento informado y se llevó a cabo la recogida de datos. Las entrevistas fueron grabadas $\mathrm{y}$ transcritas utilizando el software F4.

\section{Consideraciones éticas}

El estudio fue aprobado por el Comité de Ética de Investigación de la Universitat Internacional de Catalunya (UIC) de acuerdo con la Declaración de Helsinki. Todos los participantes firmaron el consentimiento informado y, se garantizó el anonimato y confidencialidad de los datos en base a la normativa legal vigente.

\section{Análisis de los datos}

Para el análisis de datos se utilizó desarrollo teórico propio de la Teoría Fundamentada (TF) que implicó la utilización simultánea del muestreo teórico y el método comparativo constante de datos ${ }^{29}$. En una primera fase se llevó a cabo una "codificación abierta" de los datos seguida de una "codificación focalizada" en los temas emergidos a partir de esa primera codificación sustantiva. En una segunda fase, a partir de esos códigos sustantivos iniciales, se elaboraron las categorías provisionales estableciendo sus propiedades y dimensiones dentro del proceso de "codificación axial". La elaboración del paradigma de codificación, durante todo el proceso de análisis, facilitó el establecimiento de relaciones entre las categorías emergidas como parte de la codificación selectiva, facilitando de esta manera la elaboración posterior del diálogo teorético.

\section{Criterios de rigor}

En cuanto a los criterios de rigor para asegurar la credibilidad los resultados han sido ilustrados con verbatims. Para conseguir la transferibilidad se ha detallado el contexto del muestreo. Por último, la realización de auditorías internas, dentro del equipo de investigación y externas, a cargo de un asesor externo, también ha permitido asegurar la confirmabilidad de los resultados. Para asegurar la auditabilidad de las decisiones tomadas durante todo el proceso se han ido generando toda una serie de memos ${ }^{30}$ analíticos, metodológicos y teoréticos que permiten llevar a cabo un seguimiento tanto del proceso de análisis como de la selección muestral. Por último para sistematizar y dar apoyo al proceso analítico se han utilizado estándares de trabajo y el programa informático ATLAS.ti 7.1 como soporte operativo durante todo el proceso de la investigación.

\section{Resultados y discusión}

A partir del análisis del discurso de los participantes emergieron tres categorías que dan respuesta a cuáles son los elementos que integran el significado "enfermería" para las enfermeras docentes en el caso español: Actividad humana vinculada a la maternidad, Vocación que tienen algunas personas de ayuda al otro y Actividad profesional que atiende las necesidades de los demás respetando su identidad.

\section{Actividad humana vinculada a la maternidad}

La enfermería es definida como una actividad humana vinculada a la mujer y relacionada con la maternidad, el cuidado de los hijos y la supervivencia del ser humano. Ese instinto maternal es el que proporciona la motivación y el impulso 
necesario para cuidar también de las personas enfermas y desamparadas ${ }^{31}$. Heidegger ${ }^{32}$ entiende que el cuidado, desde el punto de vista existencial, es anterior a toda actitud o situación del ser humano y surge cuando la existencia del otro adquiere importancia.

Yo creo que el hecho de que se haya vinculado el cuidado de la cría a la madre, ha permitido que evolucione, que crezca la humanidad (P4)

En relación a esto Watson \& Smith $^{33}$ en su Teoría del cuidado humano, también identifica la enfermería como un acto epistémico que contribuye a la preservación de la humanidad. Para ella cuidar es su ideal moral e implica el compromiso de proteger y realzar la dignidad humana. No obstante, los valores de las instituciones sanitarias, tanto en Estados Unidos como en otros lugares del mundo, guiados por la economía, la tecnología, la ciencia médica y la teoría administrativa no tienen en consideración lo que significa ser un ser humano, ser vulnerable y estar enfermo ${ }^{34}$. Estos modelos económicos pretenden soluciones a corto plazo centrándose en el número de camas ocupadas, en la enfermedad y el cuerpo físico como objeto tecnológico ${ }^{34}$ dejando poco espacio para el ejercicio del rol enfermero.

Esta predisposición natural hacia el cuidado determina la socialización de la mujer en ese rol en prácticamente las culturas del mundo. Para Germán esa misma atención cuidadosa, cuando se acompaña al nacer o al morir, es la que genera en la enfermera una satisfacción de plenitud humana ${ }^{35}$ que va más allá de la satisfacción profesional. Esto es identificado como una de los motivos por los cuales la enfermería es una profesión eminentemente femenina - en el caso español el 86,47 \% de egresados de las universidades son mujeres ${ }^{36}$ - pese que los hombres también presentan los atributos necesarios para poder ejercerla. En relación a esto también se identifica que en la propia disciplina médica las mujeres generalmente ocupan especialidades relacionadas con el cuidado como es el caso de la medicina de familia, la pediatría y la obstetricia ${ }^{37}$. La propia Florence Nigthingale opinaba que las mujeres médicos debían centrar más su atención a estas especialidades más vinculadas a la naturaleza femenina ${ }^{38}$.

Con la culturización se decanta mucho. ¿Entonces qué pasa?, pues que esa predisposición a la ayuda, esa reciprocidad está mucho más asentada en la mujer. Culturalmente hablando es mucho más normal que cuide una mujer (P4)

Culturalmente está muy vinculada al tema de la maternidad. Un hombre puede cuidar bien, pue- de ser muy respetuoso, puede ser muy profesional cuidando pero, es una profesión que llama menos a los hombres, por qué hace falta esa otra parte más femenina culturalmente hablando (P4)

Ese instinto natural que determina que las mujeres se decanten por llevar a cabo el rol del cuidado como parte de su naturaleza, ha favorecido la invisibilidad de la enfermería como profesión. La propia Organización Internacional del Trabajo (OIT) define las capacidades relativas al trabajo femenino como cualidades intrínsecas de la mujer no mensurables ni cuantificables y por lo tanto invisibles ${ }^{35}$. Por otro lado, el tener un rol de género orientado culturalmente al cuidado - especialmente en una sociedad familiarista como es la española ${ }^{39}$ - puede implicar priorizar la atención a la propia familia sobre la carrera profesional algo que también es identificado que sucede en las mujeres médicos ${ }^{40}$. Llegado a este punto podría ser interesante indagar si existen diferencias en cuanto a las estrategias de conciliación de la vida familiar y laboral en función del género, en el caso de la enfermería, y que efecto tiene esto sobre la promoción profesional a puestos de mayor responsabilidad y/o prestigio.

Por qué es verdad que, tradicionalmente se ha apartado a la mujer de los centros de decisión y yo creo que hay que acabar con esta situación. Pero hay situaciones que están vinculadas a la propia naturaleza de la mujer, al cuidado de la cría, el cuidado de la atención a la maternidad, a los valores femeninos (P4)

\section{Vocación de ayuda al otro que tienen algunas personas}

La enfermería también puede ser considerada una cualidad de la que disponen de forma innata algunas personas que orienta a una especial predisposición, denominada vocación, de ayuda al otro. La RAE (2016) define la vocación como "Inspiración con que Dios llama a algún estado, especialmente al de religión" y como "inclinación a un estado, una profesión o carrera" ${ }^{41}$.

$Y$ durante ese tiempo o sea, los valores asociados a lo que podríamos llamar el cuidado ¿no? Lo que entendemos la solicitud hacia los demás, esta predisposición a ayudar a los demás (P4)

Una cosa especial que te mueve por dentro, por eso defiendo la vocación (P6)

Cuando la persona toma como opción, seguir esa vocación, esto se transforma en una filosofía de vida que transciende de lo profesional a lo personal. En relación a esto la tradición socio histórica de la enfermería en España, hasta su in- 
tegración en la universidad en 1977, se ha caracterizado por la influencia de las órdenes religiosas cuidadoras donde las monjas enfermeras - y muy especialmente las Hermanas de la Caridad ${ }^{16}$ - han transmitido a las diferentes generaciones de enfermeras laicas el cuidado por "vocación"6,10,42,43. Sin embargo en el contexto anglo-americano la Reforma Protestante (siglo XVI) provocó la desaparición de las instituciones de caridad ${ }^{44}$. En este período, denominado por Donahue ${ }^{44}$ la "Etapa Oscura de la Enfermería”, los cuidados eran llevados a cabo por personas laicas procedentes de los extractos más bajos de la sociedad. Sin embargo en los países del sur de Europa donde siempre han estado presentes la órdenes religiosas dedicadas al cuidado - entre los que se encuentra España - en palabras de Germán ${ }^{16}$ la reforma de la enfermería se hace por evolución y no por revolución como hizo Nigthingale.

Con lo cual esto acaba siendo una filosofía de vida. Es una filosofía de vida vinculada a mi profesión (P5)

No es que nosotros durante ocho horas al dia pongamos nuestra vida dentro de la enfermería. Es la enfermería la que entra dentro de nuestra vida. Mirar el mundo desde el cuidado lo cambia todo (P6)

Esta filosofía de vida implica ver el mundo $y$, a las personas que habitan en él, desde una perspectiva holística y enfocada a querer atender sus necesidades a través del cuidado. Esta mirada constituye el paradigma de la enfermería que transciende todos los ámbitos de la vida más allá del ejercicio profesional.

Cuando estaba en la agencia catalana del agua trabajando como gestora también miraba así a mis empleados. Y también los miraba y veía más allá. Quizás no veía la enfermedad, por qué aquella persona estaba sana. Lo miraba a él y a su entorno y con todas sus facetas, en todas sus dimensiones, la psíquica, la física, la espiritual, etc., que le estaba preocupando, que le estaba pasando, emocionalmente como estaba (P5)

Esta forma particular que tienen las enfermeras de experimentar el mundo conlleva acompañar a las personas en situaciones vitales difíciles relacionadas con la enfermedad y con la muerte. Esta misma vivencia es la que empodera a la enfermera como persona a priorizar lo importante de la vida cambiando, como consecuencia de ello, su forma de relacionarse con el otro. En relación a esto Peplau ${ }^{45}$ define la Teoría de las relaciones interpersonales identificando la enfermería como una relación interpersonal establecida entre la enfermera y el paciente que se puede convertir en una experiencia de aprendizaje y crecimiento personal para ambos. Bajo esta visión cada encuentro es considerado una oportunidad transformadora de relación, madurez, aprendizaje y crecimiento mutuo ${ }^{46}$.

Yo tenía diecisiete años cuando empecé a estudiar. Y tuve la suerte de que mi entrada en el mundo de la enfermería fue una entrada humanista donde la persona era el centro $y$, donde la vida y la muerte, jugaban un papel tan importante que marcan tu carácter de una manera determinada (P5)

$Y$ todo esto te lleva a hacer una relativización de las cosas y a pensar (vale la vida continua, ¿no?). Hay una tragedia que necesita de ayuda para que sea menos, con un acompañamiento activo hacia la muerte en este caso. Esta tragedia personal y familiar es absolutamente compatible con la vida de las otras personas que no están viviendo esto (P5)

Ser "buena persona" es considerado un valor fundamental dentro del perfil de la enfermera en el caso español. De esta manera se identifica como muy importante socializar al estudiante como "buena persona" como requisito para transformarse en un "buen profesional".

Una de las cosas en la que yo me fundamento es que creo que lo importante es formar personas. Lo de ser enfermera casi viene sólo detrás (P7)

Todo este caminar que hemos tenido desde el punto de vista docente y de gestor, ayudar al estudiante a que no sólo aprenda la técnica si no que le ayudes aprovechando para que sea mejor persona (P7)

La visión de que la enfermera, ante todo ha de ser buena persona, es lo que impregna el significado del término "enfermería" de los valores propios de la tradición cristiana vinculados a la “vocación”. Según esta visión una "buena persona-enfermera" es aquella que es "hospitalaria", es "humilde" y tiene "espíritu de sacrificio". En relación a esto la RAE define la "humildad" como "sumisión" 47 y ésta a su vez es definida como "sometimiento a alguien (.), sometimiento al juicio de alguien (.) o subordinación manifiesta con palabras y acciones" 48 . Por otro lado el "sacrificio" es considerado como "un acto de abnegación inspirado en la vehemencia del amor" 49 y "el abnegado" aquel "que se sacrifica o renuncia a sus deseos e intereses, generalmente por motivos religiosos o de altruismo" ${ }^{50}$.

Pero tampoco tienen esta visión del cuidado que tenemos en la sociedad europea, en la sociedad occidental, más de ayuda, más de hospitalidad (P2)

Los cuidados por amor a Dios en nuestro caso han durado más tiempo de lo que han durado en 
otros sitios. Y esta vocación de que te haces enfermera por qué tienes vocación. Pero esta vocación está ligada a lo religioso tiene que ver con ser humilde, subordinada, ángel de bondad y no un profesional que tiene un conocimiento sobre el otro y le puede ayudar a alcanzar sus objetivos (P6)

Esta visión de la enfermería, como actividad humana vinculada a la maternidad y basada en los valores cristianos de "hospitalidad", "humildad" y "espíritu de sacrificio", es identificada como causa de la poca visibilidad del rol social específico de la enfermería. Este es considerado parte de la naturaleza de la persona y no resultado de una formación académica intencional. En un estudio llevado a cabo por Albar y Sivianes-Fernandez ${ }^{51}$ se evidenció que los alumnos que inician los estudios de Grado en enfermería en España siguen percibiendo la enfermería como una profesión que presta asistencia a la medicina. En otro estudio $^{52}$ llevado a cabo en este caso a nivel internacional también se demostró que la enfermera es visible como profesión fundamentalmente por su rol de asistente del médico.

Pero realmente hay otros valores como es, todo el tema de la caridad mal entendida, de humildad mal entendida, que ha hecho pues que no se visualice el trabajo de enfermería. Que no se valore el trato al considerarlo como natural a la esencia de ser mujer, a la esencia de ser religiosa (P4)

La visibilidad social de la enfermería es identificada como atributo imprescindible para conseguir la autonomía profesional. En relación a esto, la "autonomía profesional" se define como la libertad otorgada social y legalmente de autogobierno y control sobre las actividades propias de la profesión sin influencia de las fuerzas externas $^{53}$.

La autonomía se consigue a medida que la enfermera se hace visible (P2).

Son consecuencias de la práctica de la autonomía profesional el estatus profesional individual, la libertad, la autoridad, el reconocimiento de competencia por parte de los otros y la satisfacción profesional ${ }^{54}$. Llegado a este punto podría ser adecuado investigar hasta qué punto ha evolucionado la autonomía profesional de la enfermería en el contexto español, a partir de su integración en la universidad, y qué relación puede tener esto con una identidad profesional que integra los valores cristianos de humildad y sumisión.

\section{Enfermería como actividad profesional que atiende las necesidades de los demás respetando su identidad}

Como actividad profesional la enfermería se encarga de la respuesta humana que tiene la persona ante la enfermedad. Su objetivo es conseguir grados de salud mejorando la calidad de vida de las personas. Esto es identificado también como un beneficio para el propio sistema sanitario al contribuir, mediante la prevención de las secuelas y complicaciones derivadas de un mal manejo de la enfermedad, a reducir el gasto sanitario.

Yo no me ocupo de infarto de miocardio, yo aquí entro con mi rol de colaboración o interdependiente es decir, yo aquí voy a poner en marcha actividades de vigilancia y control, voy a aplicar pautas médicas y yo no soy totalmente responsable de los objetivos del paciente ante su enfermedad (P2)

Yo soy una persona competente en valorar las respuestas humanas que tienen mis pacientes, si son saludables o no saludables, en marcar una estrategia de cuidado (P2)

Yo tengo hipertensión. Pues en la medida que voy a la enfermera del barrio que me controla no me quedo 'lela'. Además hay muchos estudios que demuestran lo que la enfermera mejora y ahorra ((al sistema sanitario) (P3)

Con respecto a este punto, en el caso norteamericano, lo que contribuyó al reconocimiento de la enfermería como disciplina científica (1909), fue demostrar que los cuidados enfermeros contribuían a la reducción del gasto sanitario?. La cuestión es indagar si la enfermería española, está actualmente aportando evidencia científica sobre su contribución específica al sistema sanitario mediante el ejercicio del rol del cuidado. En relación a esto en diferentes estudios relativos a las enfermeras españolas del ámbito de la atención primaria de salud (con una mayor orientación a la promoción de la salud) se evidenció un rol profesional centrado en el tratamiento de la enfermedad ${ }^{55,56}$. Como consecuencia de ello el rol propio relativo al cuidado de la enfermería es poco visible y consecuentemente accesible para la población en ese contexto ${ }^{57}$.

Ejercer la enfermería es identificado en el caso español como el acto de atender las necesidades de los demás respetando su identidad. Esto implica hacerlo como lo haría la propia persona si estuviese en condiciones de ello. Para la enfermería, el conocimiento de la diversidad cultural es la clave que permite una mejor adecuación de las prácticas sanitarias a las necesidades de la sociedad contemporánea ${ }^{58}$ caracterizada por la globalización. 
Este segundo eje es que haremos estas substitución y cubriremos estas necesidades de tal manera que, pueda mantener su identidad. O sea lo haremos como él lo haría si pudiese hacerlo (P6)

Según Leininger ${ }^{59}$ nuestro mundo cada vez es más complejo y diverso y, las enfermeras, tienen que estar preparadas para ser competentes en el cuidado de personas de diferentes creencias y maneras de vivir. La autora define la "Enfermería Transcultural" como "el área formal de estudio y trabajo centrado en el cuidado basado en la cultura, creencias de salud o enfermedad, valores y prácticas de las personas, para ayudarlas a mantener o recuperar su salud, hacer frente a sus discapacidades o su muerte" 59 .

Por último, la enfermería es considerada una actividad profesional "extraña” en cuanto a que pretende no ser necesaria, puesto que su objetivo es el empoderamiento de la autonomía de las personas.

Pero por otro lado también somos una profesión muy extraña, por qué siempre queremos no ser necesarios (P6)

Hacerlo desde la voluntad de que el otro lo pueda hacer rápidamente sin ti, de ser substituido $y$ además hacerlo. Cuando defino el valor del cuidado digo ((el valor del cuidado es una especial sensibilidad para atender las necesidades de los demás promoviendo su autonomía) (P6)

Una profesión es una actividad cooperativa que tiene como meta interna proporcionar a la sociedad un bien específico e indispensable para su supervivencia ${ }^{60}$. No obstante, según Larson, las profesiones están explícitamente orientadas al mercado de manera que intentan el dominio intelectual y colectivo de áreas de preocupación social ${ }^{61}$, creando fuentes de valor y de poder dentro de su estructura social ${ }^{62}$. En relación a esto enfermería por un lado es una profesión que proporciona un bien específico a la sociedad, el cuidado que tiene como objetivo la supervivencia. No obstante, la propia naturaleza del servicio que ofrece, con una orientación a conseguir la autonomía del otro, puede ser un factor que determine que sea poco visible en el propio mercado.

\section{Conclusión}

Siguiendo la nomenclatura de la RAE las enfermeras que ejercen en las universidades como docentes definen la enfermería en su primera acepción cómo actividad humana vinculada a la maternidad y a la supervivencia de la especie, en su segunda acepción como vocación de ayuda al otro que tienen algunas personas y en su tercera acepción cómo actividad orientada al cuidado profesional que atiende las necesidades de los demás respetando su identidad. Por lo tanto, se puede concluir que el modelo de enfermería que se está transmitiendo en las universidades españolas integra tanto su esencia de actividad humana vinculada al género femenino - relacionada con la maternidad y la supervivencia de la especie como la necesidad de tener una especial sensibilidad, denominada "vocación", de querer ayudar al otro. Las docentes establecen como valor fundamental de la enfermería el ser una buena persona en base a los valores cristianos de "hospitalidad", "humildad" y "espíritu de sacrificio". Esto es algo que demuestra la fuerte influencia que tiene todavía la "vocación" basada en los valores cristianos como parte de la socialización de las nuevas generaciones de enfermeras.

También se puede concluir que el modelo de enfermería transmitido por las docentes españolas está en línea con las visiones y modelos teóricos propuestos por algunas enfermeras anglo-americanas: concretamente con los modelos de Watson, Peplau y Leininger ${ }^{63,64}$. Destacar que estos modelos son coherentes con la tradición sociohístorica de la enfermería en el contexto español donde la enfermera es "una mujer que cuida (.) por vocación de ayuda al otro (.) respetando su identidad". Por lo tanto el "rol de cuidado centrado en la persona" es considerado por la sociedad española algo inherente a la naturaleza de algunas personas y no parte de la formación académica intencional de una profesión. Este puede ser el motivo por el cual la RAE todavía continua definiendo a la enfermería como una profesión que tiene un "rol profesional de asistente del médico" al ser el rol de "cuidado centrado en la persona" algo que forma parte de las personas que deciden dedicarse a ello.

La principal limitación de este estudio tiene que ver con el hecho de que dos miembros del equipo de investigación son mujeres y enfermeras. Esto puede haber sesgado tanto la interpretación llevada a cabo de los datos como la orientación, influenciada por el género, de la búsqueda bibliográfica. Este sesgo se ha minimizado mediante la auditoría interna y externa por parte de miembros de otras disciplinas, tanto del proceso de análisis de los datos como de la elaboración del diálogo teorético. 


\section{Colaboradores}

OR Pedrosa, J Caïs y C Monforte-Royo trabajaron por igual de forma totalmente colaborativa.

\section{Referencias}

1. Fernandez J. Elementos que consolidan el concepto de profesión. Notas para su reflexion. Redie. Rev. Elec. Investig. Educ 2001; 3(2):24-39.

2. Rodríguez N. Manual de sociología de las profesiones. Barcelona: Publicacions i Edicions Universitat de Barcelona; 2008.

3. Santo-Tomás, M. Historia de la Enfermeria. In: Fernandez C, organizadora. Enfermería Fundamental. Barcelona: Masson; 2000. p. 3-129.

4. Sellán MC. La profesión va por dentro: elementos para una historia de la enfermeria española contemporánea. Madrid: FUDEN; 2010.

5. Nightingale F. Notas sobre enfermería: qué es y qué no es. Barcelona: Elsevier Masson; 2008.

6. Ortega G, Sanchez N. El siglo X. In: Hernandez F, organizadora. Historia de la Enfermería en España: desde la antinguedad hasta nuestros dias. Madrid: Sintesis; 1996. p. 287-323.

7. Domínguez-Alcón C. Los Cuidados y la profesión enfermera en España. Madrid: Pirámide; 1986.

8. Domínguez-Alcón C, Rodriguez JA, Miguel J. Sociología y enfermería. Madrid: Pirámide; 1983.

9. Bullough B, Bullough V. Educational Problems in a Woman 's Profession. J Nurs. Edu. 1981; 20(7):6-17.

10. García C, Martínez ML. Historia de la enfermería: evolución histórica del cuidado enfermero. Madrid: Elsevier; 2001.

11. Miro-Bonet M. Los modelos conceptuales, una estrategia de poder con implicaciones profesionales. Enferm. Clin. 2010; 20(6):360-365.

12. España. Real Decreto 2.128/1977. Disposiciones sobre la integración en la Universidad de las Escuelas de Ayudantes Técnicos Sanitarios como EScuelas Universitarias de Enfermería. Boletin Oficial del Estado 1977; 23 jul.

13. Hernández J, Moral P. Historia de la enfermería: un análisis histórico de los cuidados de enfermería. Madrid: McGraw-Hill, Interamericana de España; 1995.

14. Miró-Bonet M, Gastaldo D, Gallego-Caminero G. ¿̨Por qué somos como somos? Discursos y relaciones de poder en la constitución de la identidad profesional de las enfermeras en España (1956-1976). Enferm. Clin. 2008; 18(1):26-34.

15. España. Orden del 13 de diciembre de 1978. Disposiciones sobre la habilitación de títulos para impartir docencia. Boletín Oficial del Estado 1978; 13 dic.

16. Germán C. La revolución de las batas blancas: la enfermería española de 1976 a 1978. Zaragoza: Prensas de la Universidad de Zaragoza; 2013.

17. Real Academia de la lengua Española. Enfermeria; 2016.

18. Real Academia de la lengua Española. Enfermera/o; 2016.

19. Brito P. El camino hacia la identidad enfermera. Met. Enferm. 2007; 10(2):51-54.

20. Velasco H. Hablar y pensar, tareas culturales: temas de antropología lingüística y antropología cognitiva. Madrid: Universidad Nacional de Educación a Distancia; 2003.

21. Ramírez E. Etnicidad, identidad y migraciones: teorías, conceptos y experiencias. Madrid: Editorial Centro de Estudios Ramón Areces; 2007. 
22. Santos de Araújo L, Souza F. Consideraçoes sobre o processo de construçao da identidade do enfermeiro no cotidiano de trabalho. Rev Latino-am Enferm 2004; 12(1):50-57.

23. Borobia R. La Hipótesis en Estudios Cualitativos. El Caso de la Inducción Analítica en una Investigación sobre Adolescencia. Rev. Pilquen Sección Ciencias Soc 2004; V(6):1-12.

24. Smelter NJ, Baltes PB. Analytic Induction. International. Encyclopedia of the Social \& Behavioral Sciences. Oxford: Elsevier Science; 2001.

25. Thorne S, Kirkham SR, O'Flynn-Magee K. The Analytic Challenge in Interpretive Description. Int J Qual Methods $2004 ; 3(1): 1-11$.

26. Thorne S, Kirkham S, MacDonald-Emes J. Interpretive Description: A Noncategorical Qualitative Alternative for Developing Nursing Knowledge. Res. Nurs. Health 1997; 20(2):169-177.

27. Seidman I. Interviewing as qualitative research: a guide for researchers in education and the social sciences. New York: Teachers College Press; 2013.

28. Strauss AL, Corbin J. Basics of qualitative research: techniques and procedures for developing grounded theory. Thousand Oaks: Sage; 2015.

29. Strauss AL, Corbin J. Bases de la investigación cualitativa : técnicas y procedimientos para desarrollar la teoría fundamentada. Medellín:Universidad de Antioquia; 2002.

30. Charmaz K. Constructing grounded theory a practical guide through qualitative analysis. London: Sage; 2006.

31. Carrasco MDC, Márquez M, Arenas J. Anthropology-nursing, perspectives in gender. Cult. los Cuid. Rev. enfermería y humanidades 2005; IX(18):52-59.

32. Malvárez S. El reto de cuidar en un mundo globalizado. Texto Context. - Enferm. 2007; 16(3):520-530.

33. Watson J, Smith MC. Caring science and the science of unitary human beings: a trans-theoretical discourse for nursing knowledge development. J. Adv. Nurs. 2002; 37(5):452-461.

34. Watson, J. Caring theory as an ethical guide to administrative and clinical practices. JONAS. Healthc. Law. Ethics Regul. 2006; 8(3):87-93.

35. German C. Género y enfermería. Index Enferm. 2004; 13(46).

36. Siles J. Historia de la enfermería. Madrid: Difusión Avances de Enfermería; 2011.

37. Bell AV, Michalec B, Arenson C. The (stalled) progress of interprofessional collaboration: the role of gender. $J$. Interprof. Care 2014; 28(2):98-102.

38. MacMillan KM. The challenge of achieving interprofessional collaboration: Should we blame Nightingale? J. Interprof. Care 2012; 26(5):410-415.

39. Brullet C. Cambios familiares y nuevas políticas sociales en España y Cataluña. El cuidado de la vida cotidiana a lo largo del ciclo de vida. Educar 2010; 45: 51-79.

40. Arrizabalaga P, Abellana R, Vinas O, Merino A, Ascaso C. Women doctors and their careers in a large university hospital in Spain at the beginning of the 21st century. Hum. Resour. Health 2015; 13(1):15.

41. Real Academia de la lengua Española. Vocación; 2016.

42. Bernabeu-Mestre J. Madres y enfermeras. Demografía y salud en la política poblacionista del primer franquismo, 1939-1950. Rev. Demogr. Histórica 2002; $\mathrm{XX}(1): 123-143$
43. Sellán MC. Identidad y conflicto en el ejercicio del cuidado. Una aproximación histórica a la dinámica de la identidad enfermera en España. Madrid: Universidad Autónoma de Madrid; 2007.

44. Hernandez J. Cuestiones de enfermería. Madrid: McGraw-Hill Interamericana de España; 1996.

45. Pontes AC, Leitão I M, Ramos IC. Comunicação terapêutica em Enfermagem: instrumento essencial do cuidado. REBEn 2008; 61(3):312-318.

46. Arredondo-Gonzalez C, Siles-González J. Tecnología y Humanización de los Cuidados. Una mirada desde la Teoría de las Relaciones Interpersonales. Index Enferm. 2009; 18(1):1-7.

47. Real Academia de la lengua Española. Humildad; 2016.

48. Real Academia de la lengua Española. Sumisión; 2016.

49. Real Academica de la lengua Española. Sacrificio; 2016.

50. Real Academica d ela lengua Española. Abnegado; 2016.

51. Albar MJ, Sivianes-Fernandez M. Percepción de la identidad profesional de la enfermería en el alumnado del grado. Enferm Clin 2016; 26(3):194-198.

52. Pender S, Spilsbury K. The nursing profession: public image, self-concept and professional identity. A discussion paper. J. Adv. Nurs. 2014; 70(2):295-309.

53. Holland G. Professional nurse autonomy: concept analysis and application to nursing education. J. Adv. Nurs. 1999; 30(2):310-318.

54. Ballou K. A concept Analysis of Autonomy. J. Prof. Nurs. 1998; 14(2):102-110.

55. Corrales D, Galindo A, Escobar M, Palomo L, Magariño MJ. El debate sobre la organización, las funciones y la eficiencia de enfermería en atención primaria: a propósito de un estudio cualitativo. Aten Primaria 2000; 25(4):214-219.

56. Del Pino R, Martínez J. Estrategias para mejorar la visibilidad y accesibilidad de los cuidados enfermeros en Atención Primaria de salud. Rev. Adm. Sanit. 2007; 5(2):311-337.

57. Sancho SV, Thomàs CV, Pons RC, Pinilla MJC, Reixach JC, Montserrat MR. Análisis de situación y propuestas de mejora en enfermería de atención primaria de Mallorca: un estudio con grupos focales. Rev. Esp. Salud Publica 2002; 76(5):531-543.

58. Tarres S. El cuidado del 'otro'. Diversidad cultural y enfermería transcultural. Gaz. Antropol. 2001; 17(15).

59. Leininger M. Cuidar a los que son de culturas diferentes requiere el conocimiento y las aptitudes de la enfermería transcultural. Cult. los Cuid. 1999; III(6):5-12.

60. Hirsch A. Teoría de la educación. Salamanca: Ediciones Universidad de Salamanca; 2003

61. Rodriguez JA, Guillem M. Organizaciones y profesiones en la sociedad contemporánea. Rev. Española Investig. sociológicas 1992; 59(92):9-18.

62. Abbott A. The System of Professions: An Essay on the Division of Expert Labor. Chicago: University of Chicago Press; 1988.

63. Alligood MR, Marriner-Tomey A. Modelos y teorías en enfermería. Barcelona: Elservier Mosby; 2010.

64. Rodrigo O, Caïs J, Monforte-Royo C. Significado del término 'Enfermería' para las Enfermeras Docentes de las Universidades Españolas. Atas CIAQ2016 2008; 2:1049-1059.

Artigo apresentado em 30/01/2017

Aprovado em 25/07/2017

Versão final apresentada em 15/08/2017 